\title{
The Prevalence Onychomycosis in North Western region of Rajasthan
}

INTRODUCTION: Onychomycosis is a fungal infection of nail caused by dermatophytes, yeast and mould. Onchomycosis infection continues to spread worldwide and found to persist everywhere. Onchomycosis infection prevalence is continuously increasing and the possible fungal pathogens are also increasing. All nail diseases are not fungal in origin hence laboratory investigations are needed to differentiate accurately between fungal infections and other conditions.

AIM: to find out mycological profile \& morphological identification of fungal agent in onchomycosis infection and their prevalence in a part of Rajasthan.

MATERIALS AND METHOD: A total 50 samples of nail clipings were investigated in department of microbiology by using $20 \%$ $\mathrm{KOH}$ and culture tubes of Sabouraud's Dextrose Agar (SDA) which were mixed with chloramphenicol and cycloheximide.

RESULT: A total of 50 samples ( 18 male and 32 females) were tested in period of one year (January to December 2016). Among them $28(56 \%)$ samples showed fungal growth, the predominat fungual pathogen in present study were Tricophyton spp. 12 (42.86\%) followed by yeasts $09(32.14 \%)$ then molds $07(25 \%)$. Fungi commonly presented in the middle age, between $31-40$ years of age, due to trauma at the work site and in women, due to their wet work.

CONCLUSION: This study highlighted that Dermatophyte T.Rubrum as the main fungal pathogen which caused onychomycosis in the study region. As there are several fungi which cause the infection so it is necessary to perform culture for appropriate treatment So, it is imperative to diagnose it properly by using microbiological techniques and to treat it properly. For proper management of onychomycosis, diagnosis and accurate treatment play a key role in better outcome.

KEYWORDS: Onychomycosis, Yeasts, Dermatophytes, Middle age

\section{INTRODUCTION}

The cutenous mycoses are most common fungal infection worldwide that affect quality of life in infected patient. Dermatophytosis (tinea) infection include superficial infection of the skin, hair and nail. This infection commonly infects man and animal in developed and developing countries. Dermatophytosis does not cause mortality but it leads to morbidity. In India it is common infection due to variable topography, hot and humid climate. Dermatophytosis infection are mostly seen in summer and monsoon weather., ${ }^{1,2,3}$ The Dermatophytes spp. are mostly distributed in the temperate western countries. Onychomycosis cover $50 \%$ of the nail infections. ${ }^{4,5}$

Invasion of the nail plate by a dermatophyte is referred to as Tinea Unguium, infection of the nail by non dermatophytic fungi is called onychomycosis. Onychomycosis has been traditionally referred to unknown dermatophyte infection of the nails but is now used a general term to denote all fungal infections of nail. Onychomycosis term derived from Greek word "Onyx" a nail and "Mykes", a fungus is defined as a fungal infection of the nail. ${ }^{6,7}$ Most common cause of Onychomycosis fungal infection is dermatophytes, yeast and mould \& sometimes it is a mixed infection. ${ }^{8,9}$ Classification of Onychomycosis can be summed up as Distal Subungal Onychomycosis (DSO), White Superficial Onychomycosis (WSO), Proximal Subungal Onychomycosis (PSO) and Total Dystrophic Onychomycosis (TDO). ${ }^{10}$

In nail infection usually begins distally or at the lateral edge of the nail \& beneath the nail there is an accumulation of a cheesy epidermal detritus. The affected nails are discoloured, lustreless, brittle, thickened, friable \& may become pitted \& grooved as a result of paronychial inflammation. In some instances, the top of the nail separates distally leaving it thin, furrowed, ragged \& deformed."

Onychomycosis infection continues to spread worldwide and found persist everywhere. Onychomycosis infection prevalence is continue increasing and the possible fungal pathogens are 
also increasing. Onychomycosis prevalence rate is determined by age, predisposing factors, social class, occupation, climate \& living environment. There are many fungi which have a tendency to damage the nail, like dermatophytes (50\%), yeasts (27\%) and moulds (23\%).9,12 All the nail infection are not caused by fungus but they are also caused by other clinical conditions like trauma, wet work (with the hand submerged in water), ${ }^{13}$ HIV-AIDS, ${ }^{10}$ immunodeficiency which is due to organ or bone marrow transplantation ${ }^{10}$, old age, psoriasis, atopic dermatitis ${ }^{14}$ diabetes with a predominance of Candida spp, ${ }^{15}$ renal transplant recipients ${ }^{16}$ etc.

The fungi that commonly infect nail dermatophytes are Trychophyton rubrum, Trychophyton mentagrophytes and Epidermophyton floccosum. The yeasts that cause onychomycosis are Candida albicans, Candida parapsilosis, Candida tropicalis, etc,while the molds have long list of fungi like Scytalidium spp., Aspergillus spp., Geotrichum candidum and Fusarium spp.etc. The yeasts (candida spp.) are mainly present in the tropical and the subtropical regions and infect the persons whose hands are usually submerged in water. ${ }^{17}$ Presently in the middle age commonly found fungal agents are dermatophytes and yeasts. In the older persons over 60 years of age commonly isolated fungal agent are molds. A saprophytic fungus Scytalidium mostly present in water, soil, plants and some decaying material is transmitted by direct contact. ${ }^{8}$

In men common fungal agent for foot nail onychomycosis are dermatophytes because men have perspiration, exercise and constant wearing of shoes. In women common agent for fingernail onychomycosis are yeasts because their hands are often submerged in water.

Now a days some patients have nail disease, is a cosmetic issue. These patients do not have medical problems and regularly seek advice for cosmetic reasons. However, it can cause pain, social, emotional and occupational discomfort, permanent damage to the nail for the patient and spread of the infection to other persons. ${ }^{19}$

In last few decades, the incidence of onychomycosis is on increase due to involvement of climate, occupation, socio-economic status, gender, age and genetic and immune factors. So it is necessary to find out mycological profile \& morphological identification of fungal agent in onychomycosis infection and their prevalence.

\section{MATERIAL AND METHODS}

A prospective study was carried out on 50 patients of onychomycosis attending the department of Skin \& V.D. of P.B.M. Hospital Bikaner from January 2016 to December 2016. At the time of taking the samples patients have antifungal therapy were not included in the present study. The detailed $\mathrm{H} / \mathrm{O}$ patients was taken regarding their sociodemographic factors, presenting complaints, risk factors, predisposing factors and prior use of medication, etc. Total 50 samples of nail clippings were collected over a period in the Department of Microbiology \& Immunology, S.P. Medical College, Bikaner.

\section{Sample Collection}

The nails of the patients were wiped with $70 \%$ ethyl alcohol before the sample collection, to avoid bacterial contamination. Friable material was removed from under the nail or clipping's ans were collected from the distal border with scissors or nail clippers, where dystrophy did not extent to the distal section of the nail and scrapings was collected with a scalpel blade across the affected area.

\section{Sample processing method}

All the samples were tested in the following order: Step 1: Direct microscopy, by using freshly prepared $10 / 20 \% \mathrm{KOH}$ to identify the yeast cells, the budding yeast cells, the pseudohyphae, the hyphae and the arthroconidia.

Step 2: The culture was done in four tubes of Sabouraud's Dextrose Agar (SDA),two tubes of SDA with cycloheximide and two tubes without cycloheximide. One tube from each set was kept at $25^{\circ} \mathrm{C}$ and $37^{\circ} \mathrm{C}$ respectively. Tubes were examined twice a week for presence of growth and discarded at 4 weeks if no growth was seen.

Step 3: All the culture growths were identified on the basis of the culture characteristics, the Lactophenol Cotton Blue (LCB) test, the germ tube test, the Dalmau method by using corn meal 
agar, the sugar assimilation test and the urease test. The germ tube and the sugar assimilation tests were used to differentiate the Candida spp, while the urease test was used to differentiate T. mentagrophytes from $\mathrm{T}$. rubrum.

\section{RESULTS}

Table-1 represents the different age groups and the gender which were involved in this study. In the current study, women $(64 \%)$ had more incidence of infection than men (36\%). The mean age of the patients was 36.42 years. Most numbers of patients (34\%) were between 31-40 years of age, followed by age groups $41-50$ years $(22 \%)$ and 21 -3oyears $(20 \%)$.

A total of 50 samples of nail clippings were tested in the department of Microbiology. Culture was positive in $28(56 \%)$ cases, out of this $\mathrm{KOH}$ mount was positive in $22(44 \%)$ cases and negative in o6(12\%) cases. Culture was negative in $22(44 \%)$ cases, out of this $14(28 \%)$ cases was $\mathrm{KOH}$ positive \& $08(16 \%)$ was both culture and $\mathrm{KOH}$ negative.

Table 3 presents the fungus which were isolated from nail samples. The predominant fungus which was identified in our study was Dermatophytes in $12(42.86 \%)$ cases of the culture positive samples, which was followed by Non- dermatophyte yeast 09 (32.14\%) and molds 07(25\%). Among Dermatophytes Tricophyton species, Tricophyton rubrum showed highest incidence 06(21.42\%) cases followed by Tricophyton mantegrophytes 04(14.28\%) cases and Tricophyton interdigitae 02(07.14\%) cases. Among yeast most common isolated yeasts was Candida albicans in 05 $(17.85 \%)$ cases followed by even number of samples with Candida tropicalis 02 (07.14\%)cases \& Candida glabarata 02(07.14\%) cases. Among Non-dermatophyte moulds isolated in 07 $(25 \%)$ cases in which Aspergillus spp showed highest incidence that was isolated in $03(10.71 \%)$ cases followed by Rhizopus spp in 02 (o7.14\%)cases. Fusarium spp. and Alternaria spp. was isolated in even number o1 (03.57\%) cases.

\section{DISCUSSION}

The present study was conducted in an attempt to determine etiological agent, age, sex incidence, mycological pattern in the clinically suspected cases of onchomycosis attending the out patient of skin \& VD at P.B.M. hospital \& associated groups of hospitals, Bikaner over a period of 12 months from January 2016 to December 2016.

Recently, the incidence for onychomycosis is on increase and spectrum of causative pathogens have also increased which may be due to awareness among people towards health, cosmetic consciousness, various risk factors like chronic diseases, sport, traumatic injury and other fungal infections of the skin.

In the present study, direct microscopy was found positive in 36 cases whereas fungus was grown in 28 cases. The reason for sterile culture in cases could be that the patients were already on agents that can reduce fungal growth before the samples were taken. In our study $12 \%$ samples were identified as false negative ( $\mathrm{KOH}$ negative and culture positive). False negative findings were also reported in previous reports..$^{5,20,21}$ Thus it is fruitful to perform culture in all clinically suspected cases who are $\mathrm{KOH}$ negative as there are chances of recovering fungus in these cases on culture (being $12 \%$ in present study).

In our study we found that women have higher incidence rate $(64 \%)$ than in male (36\%), which indicate that onchomycosis was a common disease in women. Women are commonly affected because mostly women are housewives and they constantly submerge of their hands in water. This finding is similar to various other studies. . $, 9,13,22^{2}$

In present study, age was also observed as a determinantal factor in onchomycosis. Finger nail onychomycosis was commonly isolated in the middle age. Observations show clear that clinical infection of onchomycosis isolates between the ages of 31-40 years (34\%) followed by age groups 41-50 years (22\%) and $21-30 y e a r s(20 \%)$. Six cases even (12\%) found in both between $11-20$ years \& $>50$ year of age. In the middle age outdoor activity is maximum which decreases with age in elderly and old age. In young age it is also observed that repeated traumatic injuries at work sites like in farms or in industrial occupation are more and farmers have more exposure to soil, dust \& moisture. However immunity is comparatively 
stronger in young age group but more cases of onchomycosis were observed in this age group.

This shows that infection is more related to personal hygiene rather than status of immunity. We found in our study that fungal infection of nail have low incidence in older persons and teenagers. Similar observations are found by other studies. ${ }^{4,5}$

In our study we found that Dermatophytes (Tricophyton) are the most common fungal agent isolated in onychomycosis. ${ }^{16,19}$ Dermatophytes are main cause of $90 \%$ toe nail and $50 \%$ finger nail onychomycosis. ${ }^{7}$ In our study Tricophyton species were present in $12(42.86 \%)$ cases, which included o6 $(21.42 \%)$ cases with Tricophyton rubrum, 04(14.28\%) cases with Tricophyton mantegrophytes and $02 \quad(07.14 \%)$ cases Tricophyton interdigitae. Dermatophytes and yeasts are have equal incidence in some studies. ${ }^{21}$ Yeasts as a common fungal agent in now days was observed in other studies. ${ }^{16,23,24,25,22}$ These observations are similar to our study. In our study the yeasts was isolated in o9 $(32.14 \%)$ cases, in which Candida albicans in $05(17.85 \%)$ cases followed by even number of samples with Candida tropicalis 02 (07.14\%) cases \& Candida glabarata $02 \quad(07.14 \%)$ cases. The increasing incidence of yeasts was due to continuous and repeated contact with water, which is mode of transmission of Candida spp.

In our study we found Non-dermatophyte moulds in $07(25 \%)$ cases in which Aspergillus spp. was isolated in $03(10.71 \%)$ cases followed by Rhizopus spp in $02(07.14 \%)$ cases, Fusarium spp. 01(03.57\%) cases and Alternaria spp. o1 (03.57\%) cases. These studies have similar trend in other studies. ${ }^{8,9}$ Nondermatophytic molds are mostly isolated in older age. The causative cause in older age may be poor blood circulation, poor personal care, lower immune response and some systemic diseases like diabetes.

Onychomycosis is a fungal infection of finger and toe nails that affect the quality of life. Diagnosis of onychomycosis in clinically is too difficult because inappropriate collection of material for analysis \& ineffective treatment make it hard to assess the true profile of such infections., ${ }^{7,226,27}$ For proper management of onychomycosis, diagnosis and accurate treatment play a key role in better outcome. However several newer diagnostic methods have been introduced like PCR based methods and non-invasive methods like optical coherence tomography, confocal laser scan microscopy, matrix assisted laser desorption time of flight mass spectrometry (MALDI-TOF MS) and phase contrast hard $x$-ray microscopy. ${ }^{27}$ The newer technique may help in early diagnosis and better management, but the spectrum of disease is based on various etiological agents so the culture will remain gold standard in identifying the species. The main issue for such newer methods is that these facilities are available only at some higher centers. For treating onychomycosis different treatment strategies including topical, systemic and surgery are commonly used. Proper and good therapeutic response was unsatisfactory due to therapeutic failure, relapse and reinfection. ${ }^{8,12,26,27,28}$

To combat poor response newer strategies such as combination, sequential \& supplementary therapy have been suggested. The following are the strategies to improve cure rate in onychomycosis. ${ }^{28}$

- Ensure proper diagnosis.

- Choose most appropriate antifungal drug .

- Ensure bioavailability and compliance.

- Monitor for any possible drug interactions.

- Consider supplemental therapy-in poor prognostic factors.

- Consider surgery in addition to antifungal therapy if required.

- Education regarding risk factors and nail care.

- Ensure treatment of affected contacts.

- Maintain proper hygiene of hand \& foot.

\section{CONCLUSION}

Our study concludes that prevalence of onychomycosis is moderate to high in south west Rajasthan. The present study highlighted that the dermatophyte T. Rubrum was a predominant pathogen in our region. As there are several fungi which cause the infection so it is necessary to perform culture for appropriate treatment. A high frequency of fingernail onychomycosis was observed among women and so, they were advised to improve their health and personal hygiene. Females are more prone to develop onychomycosis of fingernails due to frequent contact with soap and water. The newer diagnostic methods which are rapid and accurate may 
contribute in early diagnosis and management of infection.

\section{REFERENCES}

1. Azulay RD, Azulay DR,Azulay-Abulafia L.Dermatologia. $5^{\text {th }}$ ed. Rio de Janeiro:Guanabara Koogan;2008.

2. Peres NTA, Maranhao FCA, Rossi A, MartinezRossi NM. Dermatophytes: host-pathogen interaction and antifungal resistance .An Bras Dermatol.2010;85:657-67.

3. Singh S, Beena PM. Comparative study of different microscopic techniques and culture media for the isolation of dermatophytes. Indian J Med Microbiol 2003;21:21-4.

4. Madhuri JT. Onychomycosis: A significant medical problem.Indian J Dermatol Venerol Leprol. 2002;68:326-27.

5. Veer P, Patwardhan NS, Damle AS. Study of Onychomycosis: Prevailing fungi and pattern of infection. Indian J Med Microbiol 2007;25(1):53-6.

6. Chander J. Textbook of Medical Mycology, Third Edition, New Delhi, Mehta Publishers. 2010 ;132.

7. Kaur R, Kashyap B, Bhalla P. OnychomycosisEpidemiology, Diagnosis, Management. Indian J Med Microbiol 2008;26(2);108-16.

8. Sanjiv A, Shalini M, Charoo H. Etiological agents of onychomycosis from a tertiary care hospital in Central Delhi, India. Indian J Fund Appl Life Science. 2011;1(2):11-14.

9. Gelotar P, Vachhani S,Patel B, Makwana N. The Prevalence of Fungi in Fingernail Onychomycosis. J Clin Diagn Res. 2013 ; 7(2): 250-2.

10. Torres-Rodriguez, JM, Lopez-Jodra O. Epidemiology of nail infection due to keratinophilic fungi. Rev Iberoam Micol 2000;17:122-35.

11. Emmons CW, Bindord $\mathrm{CH}$ and Utz JP. Medical Mycology, Lea and Febiger,Philadelphia,1963;86199.

12. Richardson MD, Warnock DW. Fungal Infection Diagnosis and Management. 3rd Edn. Blackwell Publishing; 2003.

13. Neupane S, Pokhrel DB, Pokhrel BM. Onychomycosis: A clinicoepidemiological study, Nepal Med Coll J. 2009; 11(2):92-95.

14. Sigurgeirsson B, Steingrimsson O. Risk Factors associated with Onychomycosis. J Eur Acad Dermatol Venereol 2004;18:48-51.

15. Qureshi N. Studying and Diagnosing the Main Causative Agent of Onychomycosis through
Laboratory Procedures. International Journal of Multidisciplinary Sciences and Engineering. 2012;3(2):13-15.

16. Szepietowski JC. Selected clinical aspects of onychomycosis. Mikol Lek 2004; 11(2):119-28.

17. Onychomycosis, From Wikipedia, free encyclopaedia. Accessed from http://en.wikipedia.org/wiki/Onychomycosis. 18. Cursi IB, Freitas LB, Neves Mde L, Silva IC. Onychomycosis by Scytalidium spp.: Clinical and epidemiological study in a university hospital in Rio de Janeiro, Brazil. An Bras Dermatol 2011 ;86(4):689-93 2011; 86 (4): 689-93.

19. Elewski BE. Onychomycosis - Pathogenesis, Diagnosis and Management. Clin Microbiol Rev. 1998 ;11(3): 415-29.

20. Das NK, Ghosh P, Das S, Bhattacharya S, Dutta $\mathrm{RN}$, Sengupta SR. A study on the etiological agent and clinico-mycological correlation of fingernail onychomycosis in eastern India. Indian J Dermatol. 2008;53:75-79.

21. Gupta M, Sharma NL, Kanga AK, Mahajan VK, Tegta GR. Onychomycosis: Clinico-mycologic study of 130 patients from Himachal Pradesh, India. Indian J Dermatol Venereol Leprol. 2007;73:389-92.

22. Brilhante RSN, Cordeiro RA, Medrano DJA, Rocha MFG, Monteiro AJ, Cavalcante CSP, Meireles TEF, Sidrim JJC. Onychomycosis in Ceará (Northeast Brazil): epidemiological and laboratory aspects. Mem Inst Oswaldo Cruz 2002;100:131-35. 23. Geramishoar M, Zomorodian K, Emami M, Tarazoei B, Saadat F. Study and Identification of the Etiological Agents of Onychomycosis in Tehran, Capital of Iran. Iranian J Publ Health 2002;31:100-4.

24. Zaini F, Mahmoudi M, Mehbod ASA, Kordbacheh P, Safara M. Fungal Nail Infections in Tehran, Iran. Iranian J Publ Health 2009;38:46-53. 25.Bramono K, Budimulja U. Epidemiology of Onychomycosis in Indonesia. Jpn J Med Mycol 2005;46(3):171-76.

26. Kaur R, Kashyap B, Bhalla P. A five-year survey of onychomycosis in New Delhi, India: Epidemiological and laboratory aspects. Indian J Dermatol 2007:52:39-42.

27. Singhal A, Khanna D. Onychomycosis: Diagnosis and management. Indian J Dermatol Venereol Leprol 2011;77(6):659-72.

28. Grover C, Khurana A. Onychomycosis: Newer insight in pathogenesis and diagnosis. Indian $\mathrm{J}$ Dermatol Venereol Leprol 2012;78(3):263-70. 
Source of support: Nil, Conflict of interest: None declared

Cite this article as:

Kumar R, Pannu S, Kumar M, Yadav OP. The Prevalence Onychomycosis in North Western region of Rajasthan. Int Healthcare Res J 2018;1(10):323-329.

\section{AUTHOR AFFILIATIONS}

1. Senior Demonstrator, Department of Clinical Microbiology \& Immunology, SP Medical College, Bikaner

2. Senior Demonstrator, Department of Clinical Microbiology \& Immunology, SP Medical College, Bikaner

3. $\mathrm{MD}$ (Microbiology)

4. Research Scientist, Department of Clinical Microbiology \& Immunology, SP Medical College, Bikaner

\section{Corresponding Author:}

\section{Dr. Sanju Pannu}

Senior Demonstrator

Department of Clinical Microbiology

\& Immunology, SP Medical College

\section{Bikaner -334001}

For manuscript enquiry/author contact details, e-mail at: maunscriptenquiry.ihrj@gmail.com

\section{LEGENDS}

\begin{tabular}{|c|c|c|c|c|c|c|}
\hline \multirow{2}{*}{ AGE } & \multicolumn{5}{c}{ FEMALE } & \\
\hline & No & $\%$ & No & $\%$ & No & $\%$ \\
\hline $11-20$ & 03 & 06 & 03 & 06 & 06 & 12 \\
\hline $21-30$ & 02 & 04 & 08 & 16 & 10 & 20 \\
\hline $31-40$ & 06 & 12 & 11 & 22 & 17 & 34 \\
\hline $41-50$ & 04 & 08 & 07 & 14 & 11 & 22 \\
\hline$>50$ & 03 & 06 & 03 & 06 & 06 & 12 \\
\hline Total & 18 & 36 & 32 & 64 & 50 & 100 \\
\hline
\end{tabular}

Table 1. Distribution of Patients According to Age Group and Gender 


\begin{tabular}{|c|c|c|c|c|}
$\begin{array}{c}\text { NAME OF } \\
\text { METHOD }\end{array}$ & OUTCOME & No. & $\begin{array}{c}\text { FUNGUS CULTURE } \\
\text { POSITIVE }\end{array}$ & $\begin{array}{c}\text { FUNGUS CULTURE } \\
\text { NEGATIVE }\end{array}$ \\
\hline KOH Mount & Positive & $36(72 \%)$ & $\mathbf{2 2}(44 \%)$ & $\mathbf{1 4}(\mathbf{2 8} \%)$ \\
& Negative & $\mathbf{1 4}(\mathbf{2 8 \% )}$ & o6(12\%) & o8(16\%) \\
\hline Total Cases & & $\mathbf{5 0}$ & $\mathbf{2 8}(\mathbf{5 6 \% )}$ & $\mathbf{2 2}(\mathbf{4 4} \%)$ \\
\hline
\end{tabular}

Table 2. Correlation between the Findings of Direct Microscopy (KOH) and Fungal Culture

\begin{tabular}{|c|c|c|c|c|}
\hline CATEGORY & FUNGAL SPP. & NUMBERS & PERCENTAGE $(\mathrm{N}=\mathbf{2 8})$ & $\mathrm{N}=\mathbf{2 8}$ \\
\hline Yeasts & $\begin{array}{l}\text { C.albicans } \\
\text { C.tropicalis } \\
\text { C.glabarata }\end{array}$ & $\begin{array}{l}05 \\
02 \\
02\end{array}$ & $09(32.14 \%)$ & $\begin{array}{l}17.85 \% \\
07.14 \% \\
07.14 \%\end{array}$ \\
\hline Dermatophytes & $\begin{array}{c}\text { T.rubrum } \\
\text { T.mentagrophytes } \\
\text { T.interdigitae }\end{array}$ & $\begin{array}{l}06 \\
04 \\
02\end{array}$ & $12(42.86 \%)$ & $\begin{array}{l}21.42 \% \\
14.28 \% \\
07.14 \%\end{array}$ \\
\hline Molds & $\begin{array}{c}\text { Aspergillus Spp. } \\
\text { Rhizopus spp } \\
\text { Fusarium } \\
\text { Alternaria }\end{array}$ & $\begin{array}{l}03 \\
02 \\
01 \\
01\end{array}$ & $07(25 \%)$ & $\begin{array}{l}10.71 \% \\
0.7 .14 \% \\
03.57 \% \\
03.57 \%\end{array}$ \\
\hline
\end{tabular}

Table 3. Distribution of Fungus 